\title{
miR-223 promotes cartilage differentiation of bone marrow-derived mesenchymal stem cells and protects against osteoarthritis by suppressing NLRP-3 expression
}

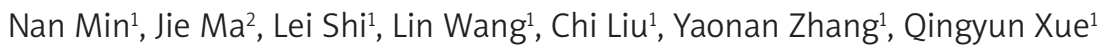

${ }^{1}$ Department of Orthopedics, Beijing Hospital, Beijing, China

${ }^{2}$ Center of Biotherapy, Beijing Hospital, National Center of Gerontology;

Institute of Geriatric Medicine, Chinese Academy of Medical Sciences, Beijing, China

Submitted: 3 August 2020

Accepted: 5 September 2020

Arch Med Sci

DOI: https://doi.org/10.5114/aoms.2020.100640

Copyright (c) 2020 Termedia \& Banach

\begin{abstract}
Introduction: The present investigation evaluates the role of miR-223 mimic in the treatment of osteoarthritis (OA) and postulates the possible molecular mechanism of its action.

Material and methods: Bone marrow-derived mesenchymal stem cells (BM$\mathrm{SCs}$ ) were isolated from rats and cultured in chondrogenic medium to stimulate the differentiation of chondrocytes. Alcian blue staining was performed to determine the chondrogenic differentiation and expression of miR-223 in the BMSCs. Moreover, expression of NLR family pyrin domain containing 3 (NLRP-3), matrix metallopeptidase-13 (MMP-13) and collagen (Col II) was determined in miR-223 mimic and inhibitor treated BMSCs. OA was induced by injecting anterior cruciate ligament transection in rats followed by further treatment with the miR-223 mimic for the period of the treatment protocol. Level and expression of inflammatory cytokines were estimated in the cartilage tissue of OA rats. Moreover, immunohistochemical analysis and histopathology study were also performed.

Results: Data of the study reveal that expression of miR-223 was higher in chondrogenic differentiated BMSCs than normal. Expression of MMP-13 and NLRP-3 was lower, and expression of Col II was higher in miR-223 mimic treated BMSCs than normal. Moreover, data of the in-vivo study indicate that the expression level of cytokines was lower in the cartilage tissue of the miR-223 mimic treated group than the OA group. Treatment with the miR223 mimic ameliorates the altered histopathology and expression of NLRP-3 in the cartilage tissue of OA rats.

Conclusions: Data of the study reveal that the miR-223 mimic enhances the chondrogenic differentiation of BMSCs by regulating the NLRP-3/IL-18/TGF- $\beta$ pathway.
\end{abstract}

Key words: miR-223, osteoarthritis, bone marrow-derived mesenchymal stem cells, cartilage, inflammation.

\section{Introduction}

Osteoarthritis (OA) is clinically the most common form of arthritis, associated with articular cartilage damage and degradation [1]. OA aetiologies include abnormal loading, repetitive injuries, ageing, and obesity [2];
Corresponding author:

Qingyun Xue PhD

Department of Orthopedics

Beijing Hospital

100730 Beijing, China

E-mail: cramen2006@163.com 
OA increases socioeconomic burdens worldwide, including China [3]. OA is associated with physical disabilities and joint pain. Several degradative pathological changes are evident in cartilage [4]; and chondrocytes undergo apoptosis [5]. Both osteoblasts and chondrocytes are formed via differentiation of bone marrow-derived mesenchymal stem cells (BMSCs) [6]. Such cells are used to manage olfactory disorders, pelvic floor dysfunction, and pulmonary injury caused by ischaemia. BMSCS regenerate cartilage in animal models of $\mathrm{OA}$; the cells are simple to isolate, multipotent, and highly proliferative [7]. BMSCs ameliorate $\mathrm{OA}$-induced cartilage injury by differentiating into chondrocytes.

miRNA dysregulation is linked to the development of several diseases, including $\mathrm{OA}$, and the miRNAs may serve as useful therapeutic targets [8]. miRNA dysregulation induces the production of inflammatory mediators, remodelling of the extracellular matrix, and dysregulation of bone formation [9]. miR-223 expression slows the cartilage degeneration of OA and rheumatoid arthritis [10]. Several molecules ameliorate cartilage degeneration by enhancing miR-223 expression and reducing that of NLRP3 [11], thus lowering inflammatory cytokine levels [12]. NLRP3 expression is downregulated by miR-223-3p; we thus evaluated the effect of miR-223-3p on the chondrocytic differentiation of BMSCs.

\section{Material and methods}

\section{Animals}

Sprague-Dawley rats (200-250 g) were housed and maintained as per international guidelines; the animal study was approved by the Animal Ethics Committee of Beijing Hospital, China (no. IAEC/ $\mathrm{BH} / 2018 / 09$ ).

\section{Chemicals}

The miR-223 inhibitor and mimic were procured from Invitrogen (USA). The ELISA kits and primers were the products of Cell Signaling Technology Inc. (USA).

\section{Chondrocyte isolation and culture}

Primary BMSCs were isolated as described previously [13] from the cavities of the tibial plateaus and femora and cultured for 2 days at $37^{\circ} \mathrm{C}$ in Dulbecco's modified Eagle's medium (DMEM) supplemented with transforming growth factor- $\beta 3$ (TGF- $\beta$ ), sodium pyruvate, ascorbate, and dexamethasone. Non-adherent cells were removed during medium changes, which were performed until the cells attained $80-90 \%$ confluence. Flow cytometry was used to characterise the BMSCS in terms of typical surface markers. Antibodies against CD29, CD90, CD45, or CD105 were added to BMSCs following the manufacturer's directions.

\section{Alcian blue staining}

BMSCs were cultured for 0,14 , and 21 days in chondrogenic or normal medium and fixed in $4 \%$ $(\mathrm{v} / \mathrm{v})$ formalin at $4^{\circ} \mathrm{C}$; Alcian blue $8 \mathrm{GX}(1 \% \mathrm{v} / \mathrm{v})$ was added and staining proceeded for $60 \mathrm{~min}$ at room temperature. The cells were washed with water and a flatbed scanner was used to detect signals. Staining intensity was quantified with the aid of Image-Pro Plus software.

\section{RT-PCR}

Trizol reagent was used to extract total RNAs, followed by reverse transcription into cDNAs. The levels of miR-223 and $\beta$-actin expression in BMSCs were derived with the aid of the SYBR Green gene expression assay. miR-223 levels were also analysed using a TaqMan microRNA assay kit (Table I).

\section{Cell transfection}

Isolated cells were seeded into 12 -well plates and incubated for 1 day at $37^{\circ} \mathrm{C}$ in medium with Lipofectamine 2000. The miR-223 inhibitor or mimic was added, and incubation continued for a further day. Control cells were not transfected.

\section{In-vivo model of osteoarthritis}

The animals were divided into normal, OA, and miR-223 mimic-treated groups. All were injected with $7 \mathrm{mg} / \mathrm{kg}$ xylazine and $60 \mathrm{mg} / \mathrm{kg}$ of ketamine. To induce OA, the medial side of the patellar tendon was incised, the patella dislocated, and the anterior cruciate ligament transected with a surgical blade. The medial retinaculum was repaired, and the skin closed. The miR-223 mimic was delivered directly to the articular cavity. qRT-PCR was performed (as mentioned above).

Table I. The primers shown in this study

\begin{tabular}{|lcc|}
\hline Primers & Forward & Reverse \\
\hline miR-223 & CGTGTATTTGACAAGCTG & GAACATGTCTGCGTATCTC \\
\hline$\beta$-actin & CACCATTGGCAATGAGCGGTTC & AGGTCTTTGCGGATGTCCACGT \\
\hline
\end{tabular}


Table II. The primers

\begin{tabular}{|c|c|c|}
\hline Primers & Forward & Reverse \\
\hline NLRP-3 & 5'-AAAGCCAAGAATCCACAGTG TAAC-3' & 5'-TTGCCTCGCAGGTA AAGGT-3' \\
\hline IL-18 & 5'-GATAGCCAGCCTAGAGGTATGG-3' & 5'-CCTTGATGTTATCAGGAGGATTCA-3' \\
\hline MMP-3 & 5'-CACTCACAGACCTGACTCGGTT-3' & 5'-AAGCAGGATCACAGTTGGCTGG-3' \\
\hline Collagen II & 5'-CCTGGCAAAGATGGTGAGACAG-3' & 5'-CCTGGTTTTCCACCTTCACCTG-3' \\
\hline GAPDH & 5'-ACAG GGGAGGTGATAGCATT-3' & 5'-GACC AAAAGCCTTCATACATCTC-3' \\
\hline
\end{tabular}

\section{Estimation of protein and cytokine levels}

All animals were sacrificed via decapitation and articular cartilage isolated from the medial tibial plateaus. qRT-PCR was used to quantify the levels of mRNAs encoding NLRP-3, IL-18, MMP-3, and Col II in tissue homogenates. IL-1 1 , IL-6, IL-18, TGF- $\beta$, and NF-kB levels were measured by ELISA (Table II).

\section{Histopathological analyses}

Isolated knee joints were fixed in $10 \%(\mathrm{v} / \mathrm{v})$ formalin for 3 days, placed into molten paraffin, and cut into $6 \mu \mathrm{m}$-thick sections when the paraffin set. The sections were stained with Safranin-O and cartilage degeneration scored as described previously.

\section{Immunohistochemical analysis}

The subchondral bone was sectioned after embedding in paraffin wax. Ethanol baths were used to dehydrate the sections, which were then incubated with an anti-rat NLRP3 antibody at $4^{\circ} \mathrm{C}$ overnight. Biotinylated IgG was added and incubation continued for $30 \mathrm{~min}$ at $37^{\circ} \mathrm{C}$. The sections were then stained with diaminobenzidine (DAB) and cells counted under an optical microscope.

\section{Statistical analysis}

All data are expressed as means \pm standard errors of the means (SEMs). Statistical analysis featured one-way analysis of variance (ANOVA). Post-hoc comparisons were performed using the Dunnett test of GraphPad Prism ver. 6.1 software (USA). A $p$-value $<0.05$ was taken to indicate statistical significance.

\section{Results}

\section{BMSC characterisation}

BMSCs were assessed via flow cytometry (Figures 1 A-D). The cells were CD29-, CD90-, and CD105-positive and CD45-negative, indicating that they were BMSCs.

\section{miR-223 expression by BMSCs}

miR-223 expression during BMSC chondrogenic differentiation was assessed as shown in Figures $2 \mathrm{~A}$ and B. The extent of BMSC differentiation on days 0,14 , and 21 was evaluated by staining glycosaminoglycan with Alcian blue (Figure 2 A). Staining increased significantly by 14 and 21 days when cells were cultured in chondrogenic compared to normal medium. qRT-PCR revealed that miR-223 expression was upregulated in cells growing in chondrogenic medium (compared to normal medium) on days 14 and 21.

\section{Effects of miR-223 mimic and inhibitor on expression levels of NLRP-3, MMP-13, and Col II}

We used Western blotting to measure the expression levels of NLRP-3, MMP-13, and Col II in chondrogenically differentiated BMSCs treated with the miR-223 mimic and the inhibitor (Figure 3). The miR-223 mimic significantly $(p<0.01)$ downregulated the expression of NLRP-3, MMP-13, and Col II, compared to controls. The miR-223 inhibitor significantly $(p<0.01)$ upregulated the expression levels of all three proteins.

\section{Effects of miR-223 mimic on levels of inflammatory cytokines}

The levels of IL-1 $\beta$, IL- 6 , IL-18, NF- $\kappa B$, and TGF- $\beta$ were determined in miR-223 mimic-treated cartilage of rats with OA (Figure 4). The levels were higher $(p<0.01)$ in rats with OA than normal rats. Treatment with the miR-223 mimic reduced $(p<0.01)$ the cytokine levels.

\section{Effects of miR-223 mimic on expression of mRNAs encoding NLRP-3, IL-18, MMP-3, and Col II}

Figure 5 shows the effects of the miR-223 mimic on the levels of mRNAs encoding NLRP-3, IL-18, MMP-3, and Col II in cartilage of rats with OA. All mRNAs were expressed at lower levels than 
A

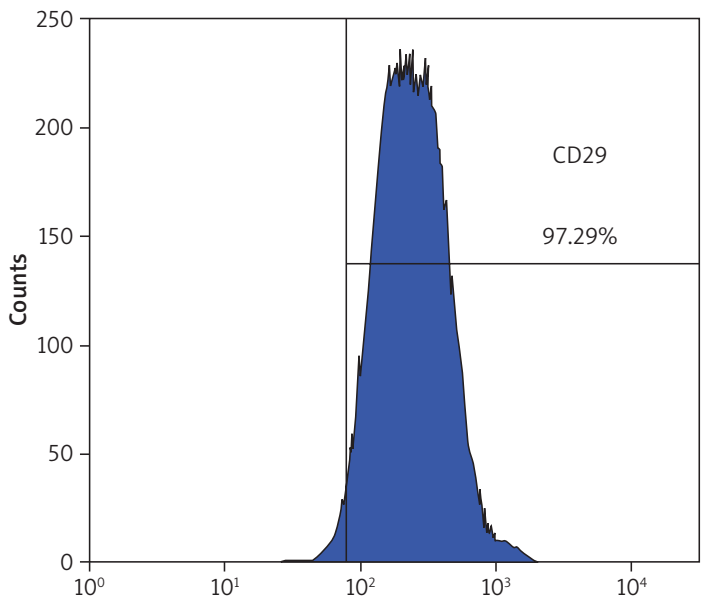

C

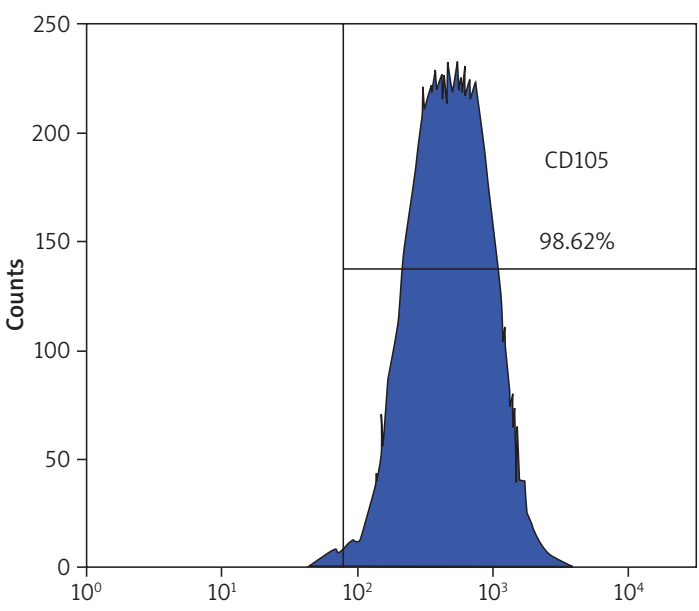

B

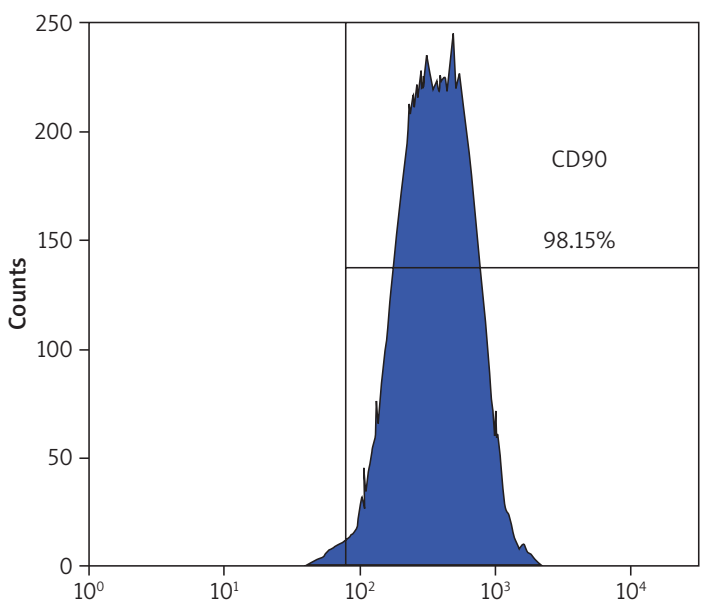

D

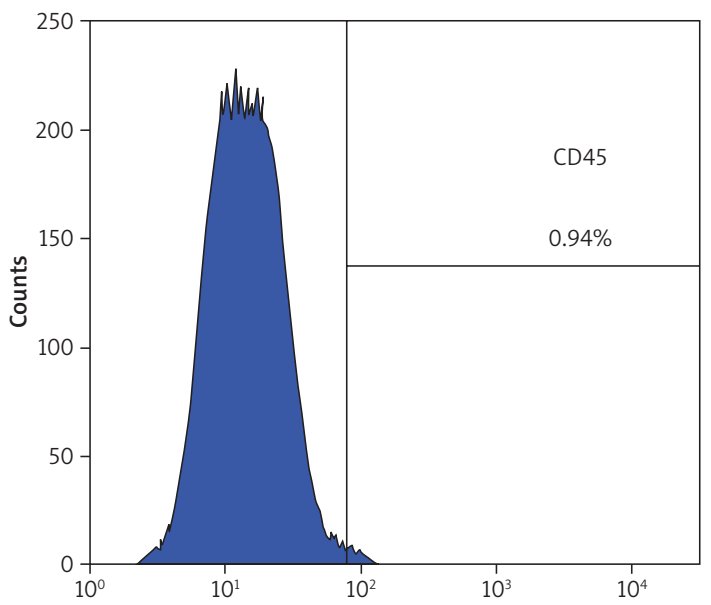

Figure 1. Quality control of bone marrow-derived mesenchymal stem cells (BMSCs). The cells were incubated with antibodies against CD29 (A), CD90 (B), CD105 (C), and CD45 (D)

in normal rats. Treatment with the miR-223 mimic significantly $(p<0.01)$ enhanced the expression levels of all tested mRNAs.

\section{Effect of miR-223 mimic on histopathology of cartilage tissue}

We stained the cartilage of miR-223 mimictreated rats with $\mathrm{OA}$ with $\mathrm{H} \& \mathrm{E}$ and safranin $\mathrm{O} /$ Fast Green (Figure 6). Cartilage erosion was more severe in the OA than the normal group. The miR223 mimic attenuated erosion.

\section{Effect of miR-223 mimic on NLRP-3 expression}

We immunohistochemically explored the effect of the miR-223 mimic on NLRP-3- positive cell numbers in the cartilage of rats with OA (Figure 7). The numbers were significantly higher $(p<0.01)$ than in normal rats. The miR-223 mimic reduced the proportion of NLRP-3- positive cells in cartilage $(p<0.01)$.

\section{Discussion}

Osteoarthritis is a major form of arthritis. Several drugs are available, but chronic OA requires chronic treatment. The drugs have several limitations; new therapies are required. miR-223 is expressed in healthy chondrocytes but the levels fall in degenerating chondrocytes. miR-223 controls NLRP-3 expression and thus, in turn, the levels of cytokines that contribute to the chondrocyte destruction characteristic of OA. We explored whether miR-223 protected against OA.

In OA, chondrocytes are damaged by foreign body intrusions, inflammation, and excessive joint loading [14]. Chondrocytes do not self-heal or regenerate, and proliferation is inhibited by the reduced cell density [15]. Management focuses on 

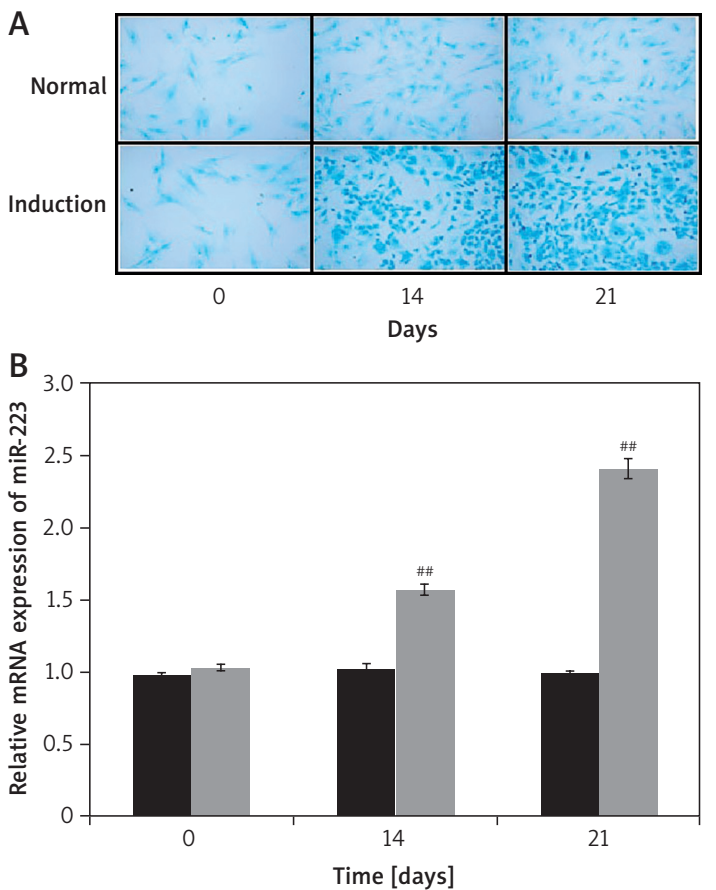

Normal Induction

Figure 2. miR-223 expression during chondrogenic BMSC differentiation. A - Assessment of chondrogenic differentiation via Alcian blue staining, $\mathbf{B}-$ miR-223 expression in chondrogenically differentiated cells

Means \pm SEMs $(n=6) ;{ }^{\# \#} p<0.01$ compared to normal group.

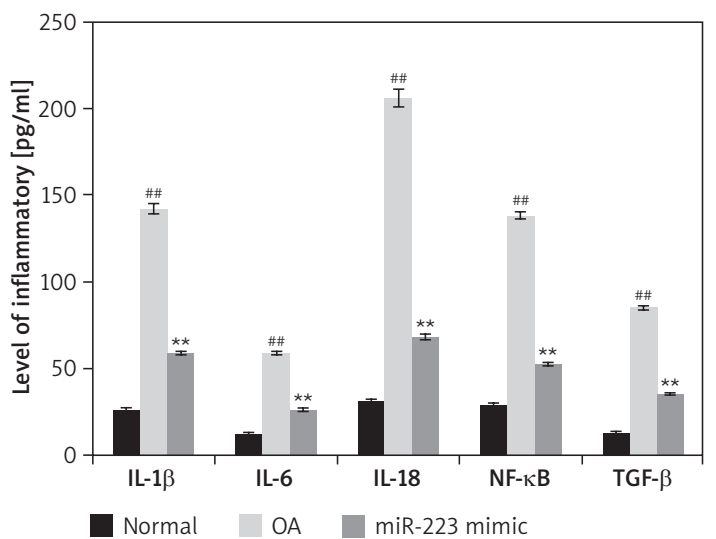

Figure 4. Effects of miR-223 mimic on levels of IL-1 $\beta$, IL-6, IL-18, NF- $\kappa B$, and TGF- $\beta$ in cartilage of osteoarthritic rats, as revealed by ELISA

Means \pm SEMs $(n=8)$; \# $p<0.01$ compared to normal group; ${ }^{* *} p<0.01$ compared to OA group.

cartilage engineering; regeneration is inefficient [16]. BMSCs differentiate into myocytes, osteoblasts, and chondrocytes [17]. Several genes are involved in chondrogenesis. miR-223 enhanced the chondrogenic differentiation of BMSCs [18] and

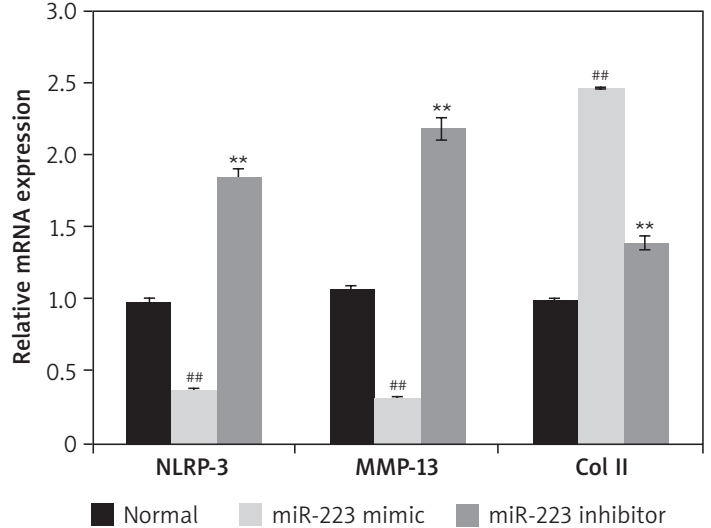

Figure 3. Effects of miR-223 mimic and inhibitor on expression levels of NLRP-3, MMP-13, and Col II in chondrogenically differentiated BMSCS

Means \pm SEMS $(n=6) ;{ }^{\# \#} p<0.01$ compared to normal group; ${ }^{* *} p<0.01$ compared to OA group.

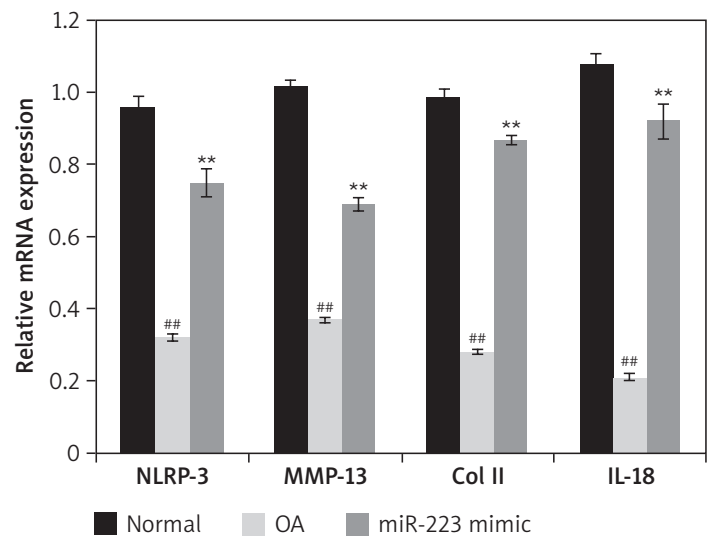

Figure 5. Effects of miR-223 mimic on levels of mRNAs encoding NLRP-3, IL-18, MMP-3, and Col II in cartilage of osteoarthritic rats, measured via qRT-PCR Means \pm SEMs $(n=8) ;{ }^{\#} p<0.01$ compared to normal group; ${ }^{* *} p<0.01$ compared to OA group.

alleviated OA chondrocyte destruction [19]. We found that miR-223 enhanced the chondrogenic differentiation of BMSCs.

NLRP-3, MMP-13, and Col II facilitate BMSC differentiation [20] toward chondrocytes [21]. In OA, the MMP-13 level is reduced and that of Col II enhanced in damaged chondrocytes [22]. MMP-13 first enhances and then reduces Col-II expression in chondrogenically differentiated BMSCs [23]. miR-223 increased NLRP-3 expression in such cells. The miR-223 mimic reduced NLRP-3 expression by BMSCs.

Cartilage destruction in $\mathrm{OA}$ is attributable to increased cytokine levels (including that of IL-18) [24] caused by a reduced level of NLRP-3. The cytokines reduce the expression levels of MMP-13, Col II, and TGF- $\beta$, all of which stimulate chondro- 


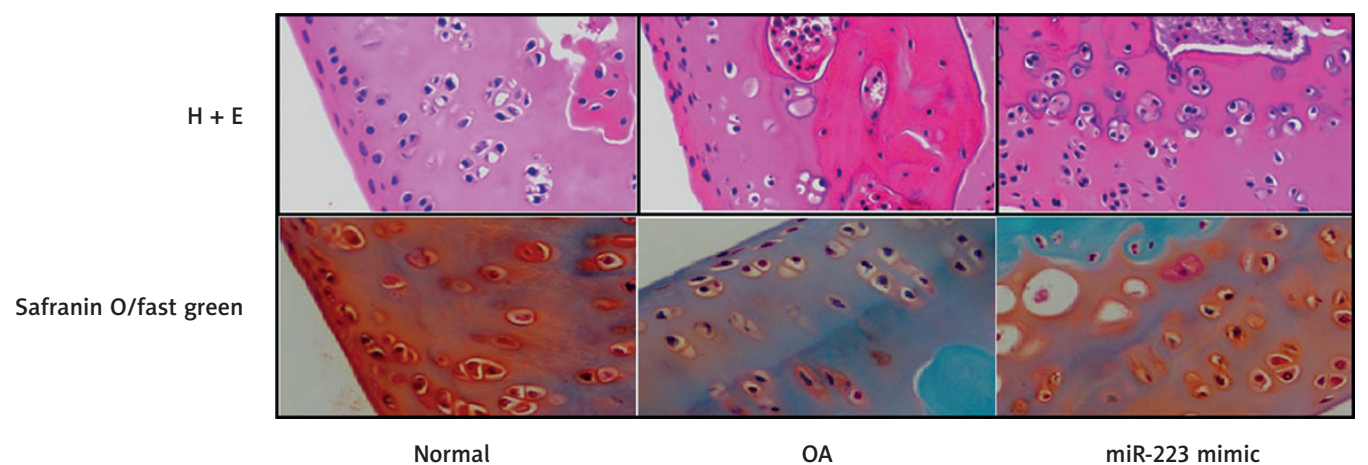

Figure 6. Effects of miR-223 mimic on cartilage histopathology in osteoarthritic rats. H\&E and Safranin O/Fast Green staining

A
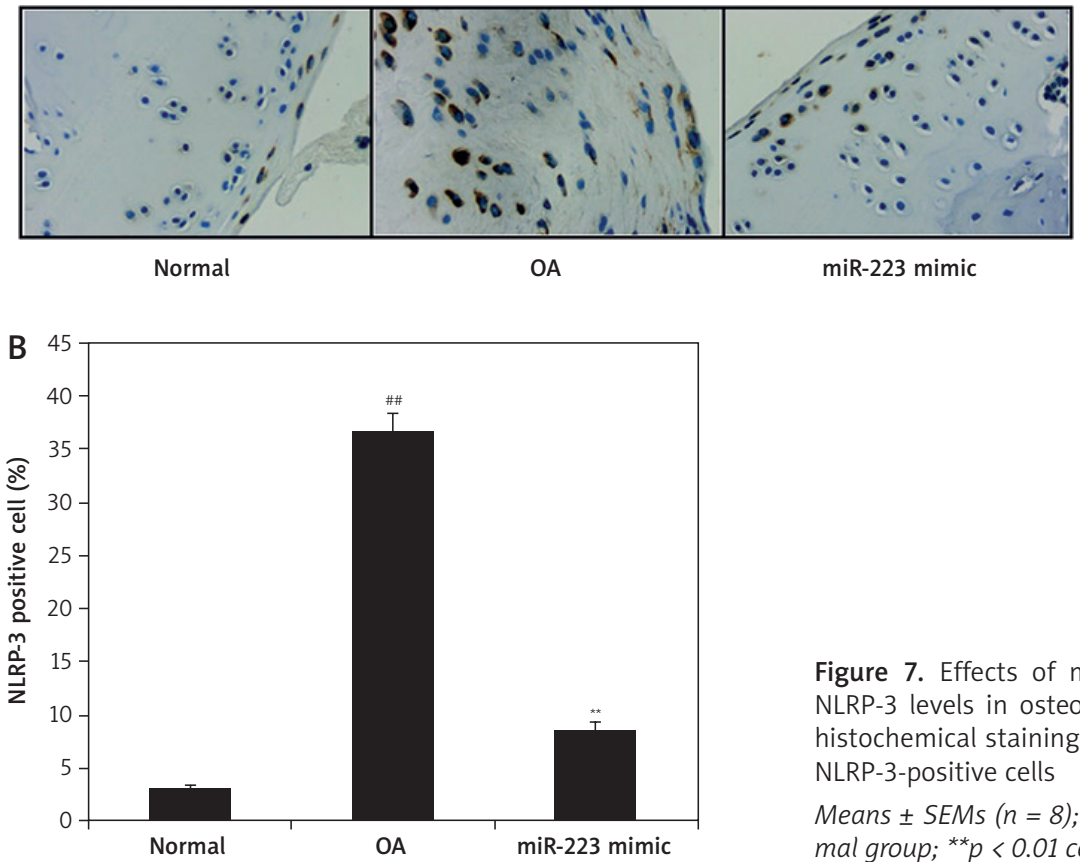

Figure 7. Effects of miR-223 mimic on cartilage NLRP-3 levels in osteoarthritic rats. A - Immunohistochemical staining for NLRP-3, B - numbers of NLRP-3-positive cells

Means \pm SEMs $(n=8) ; \# p<0.01$ compared to normal group; ${ }^{* *} p<0.01$ compared to OA group.

cyte differentiation $[25,26]$. The miR-223 mimic reduced cytokine expression, and increased the levels of NLRP-3, MMP-13, Col II and TGF- $\beta$, in the cartilage of rats with $\mathrm{OA}$.

In conclusion, we found that the miR-223 mimic enhanced the chondrogenic differentiation of BMSC $s$ by regulating the NLRP-3/IL-18/TGF- $\beta$ pathway. The mimic may thus usefully manage OA.

\section{Acknowledgments}

All authors thank the Fundamental Research Fund for the Central Universities (grant no. 3332018176) for support.

\section{Conflict of interest}

The authors declare no conflict of interest.

\section{References}

1. Man GS, Mologhianu G. Osteoarthritis pathogenesis a complex process that involves the entire joint. J Med Life 2014; 7: 37-41.

2. Shane Anderson A, Loeser RF. Why is osteoarthritis an age-related disease? Best Pract Res Clin Rheumatol 2010; 24: $15-26$.

3. Litwic A, Edwards MH, Dennison EM, Cooper C. Epidemiology and burden of osteoarthritis. Br Med Bull 2013; 105: 185-99.

4. Loeser RF, Goldring SR, Scanzello CR, Goldring MB. Osteoarthritis: a disease of the joint as an organ. Arthritis Rheum 2012; 64: 1697-707.

5. Hwang HS, Kim HA. Chondrocyte apoptosis in the pathogenesis of osteoarthritis. Int J Mol Sci 2015; 16: 26035-54.

6. Solchaga LA, Penick KJ, Welter JF. Chondrogenic differentiation of bone marrow-derived mesenchymal stem cells: tips and tricks. Methods Mol Biol 2011; 698: 253-78. 
7. Kristjánsson B, Honsawek S. Mesenchymal stem cells for cartilage regeneration in osteoarthritis. World J Orthop 2017; 8: 674-80.

8. Sondag GR, Haqqi TM. The role of microRNAs and their targets in osteoarthritis. Curr Rheumatol Rep 2016; 18: 56.

9. Malemud CJ. MicroRNAs and osteoarthritis. Cells 2018; 7: 92.

10. Rousseau JC, Millet M, Croset M, Sornay-Rendu E, Borel O, Chapurlat R. Association of circulating microRNAs with prevalent and incident knee osteoarthritis in women: the OFELY study. Arthritis Res Ther 2020; 22: 2.

11. Tezcan G, Martynova EV, Gilazieva ZE, McIntyre A, Rizvanov AA, Khaiboullina SF. MicroRNA post-transcriptional regulation of the NLRP3 inflammasome in immunopathologies. Front Pharmacol 2019; 10: 451.

12. Sokolove J, Lepus CM. Role of inflammation in the pathogenesis of osteoarthritis: latest findings and interpretations. Ther Adv Musculoskelet Dis 2013; 5: 77-94.

13. Zhang W, Zhang F, Shi H, et al. Comparisons of rabbit bone marrow mesenchymal stem cell isolation and culture methods in vitro. PLoS One 2014; 9: e88794.

14. Guilak F. Biomechanical factors in osteoarthritis. Best Pract Res Clin Rheumatol 2011; 25: 815-23.

15. Tiku ML, Sabaawy HE. Cartilage regeneration for treatment of osteoarthritis: a paradigm for nonsurgical intervention. Ther Adv Musculoskelet Dis 2015; 7: 76-87.

16. Zhang L, Hu J, Athanasiou KA. The role of tissue engineering in articular cartilage repair and regeneration. Crit Rev Biomed Eng 2009; 37: 1-57.

17. Han Y, Li X, Zhang Y, Han Y, Chang F, Ding J. Mesenchymal stem cells for regenerative medicine. Cells 2019; 8: 886.

18. Guan X, Gao Y, Zhou J, et al. miR-223 regulates adipogenic and osteogenic differentiation of mesenchymal stem cells through a C/EBPs/miR-223/FGFR2 regulatory feedback loop. Stem Cells 2015; 33: 1589-600.

19. Endisha H, Rockel J, Jurisica I, Kapoor M. The complex landscape of microRNAs in articular cartilage: biology, pathology, and therapeutic targets. JCI Insight 2018; 3: e121630.

20. Li H, Wang D, Yuan Y, Min J. New insights on the MMP-13 regulatory network in the pathogenesis of early osteoarthritis. Arthritis Res Ther 2017; 19: 248.

21. Eo SH, Kim SJ. Rosmarinic acid induces rabbit articular chondrocyte differentiation by decreasing matrix metalloproteinase-13 and inflammation by upregulating cyclooxygenase-2 expression. J Biomed Sci 2017; 24: 75.

22. Young DA, Barter MJ, Wilkinson DJ. Recent advances in understanding the regulation of metalloproteinases. F1000Res 2019 Feb 18; 8: F1000 Faculty Rev-195.

23. Wang JJ, Huan SK, Hsieh KH, et al. Inhibitory effect of midazolam on MMP-9, MMP-1 and MMP-13 expression in PMA-stimulated human chondrocytes via recovery of NF-kappaB signaling. Arch Med Sci 2013; 9: 332-9.

24. Man GS, Mologhianu G. Osteoarthritis pathogenesis a complex process that involves the entire joint. J Med Life 2014; 7: 37-41.

25. Goldring MB, Otero M, Tsuchimochi K, ljiri K, Li Y. Defining the roles of inflammatory and anabolic cytokines in cartilage metabolism. Ann Rheum Dis 2008; 67 Suppl 3(03): iii75-82.

26. Eken MK, Ersoy GS, Kaygusuz El, et al. Etanercept protects ovarian reserve against ischemia/reperfusion injury in a rat model. Arch Med Sci 2019; 15: 1104-12. 